\title{
N-Terminal Pro-Brain Natriuretic Peptide, Cardiac and Vascular Function in Patients with Type 1 Diabetes Mellitus during the Ascent of Mt. Kilimanjaro
}

\author{
Suzanna T. de Vries ${ }^{1 *}$, Pieter de $\mathrm{Mol}^{2}$, Bert D. Dikkeschei ${ }^{3}$, Marion J. Fokkert ${ }^{3}$, Hans G. Krabbe ${ }^{3}$, Eelco \\ JP. de Koning ${ }^{4}$, Rijk OB. Gans ${ }^{5}$ and Henk JG. Bilo ${ }^{5,6}$ \\ ${ }^{1}$ Department of Cardiology, Tjongerschans Hospital, Heerenveen, Netherlands \\ ${ }^{2}$ Department of Internal Medicine, University Medical Centre St. Radboud, Nijmegen, Netherlands \\ ${ }^{3}$ Department of Clinical Chemistry, Isala Klinieken, Zwolle, Netherlands \\ ${ }^{4}$ Department of Nephrology, University Medical Centre, Leiden, Netherlands \\ ${ }^{5}$ Department of Internal Medicine, University Medical Centre, Groningen, Netherlands \\ ${ }^{6}$ Department of Internal Medicine, Isala Klinieken, Zwolle, Netherlands
}

Received: July 30, 2014; Accepted: September 15, 2014; Published: October 08, 2014

*Corresponding author: Suzanna T. de Vries, Department of Cardiology, Tjongerschans Hospital, PO Box 10500, 8440 MA Heerenveen, Netherlands, Tel: 003-165-491-7434; E-mail: suzanna.de.vries@tjongerschans.nl

\begin{abstract}
Aim: Hypobaric hypoxia during high altitude mountaineering and trekking triggers physiological changes in the cardiovascular and pulmonary systems that optimize the delivery of oxygen to tissues. As Type 1 Diabetes Mellitus (T1DM) is associated with increased cardiovascular risk, our goal was to investigate any disproportionately adverse effects of hypobaric hypoxia on cardiac and vascular parameters in patients with T1DM during an expedition to the summit of Mt. Kilimanjaro.
\end{abstract}

Methods: Sixteen well-trained subjects, 8 with T1DM and 8 nondiabetic controls, climbed Mt Kilimanjaro (5,895 m) over a 7-day period. Cardiac function was evaluated by echocardiography and measurement of NTproBNP levels both at Sea Level (SL) and at 4000 $\mathrm{m}$. Vascular measurements were performed at $1300 \mathrm{~m}$ and 4000 $\mathrm{m}$, and included assessment of blood pressure (Dinamap), derived central aortic systolic pressure (B-PRO) and pulse wave velocity (VICORDER). Oxygen saturation ( $\mathrm{SatO}_{2}$ ) was assessed at $4000 \mathrm{~m}$. The Lake Louise Acute Mountain Sickness (AMS) score was obtained daily.

Results: All participants reached the summit. At $4000 \mathrm{~m} \mathrm{Sat} \mathrm{O}_{2}$ was reduced in both groups. NTproBNP levels were significantly higher in the total group at $4,000 \mathrm{~m}$ compared to SL. Left ventricular function and right ventricular diameter did not change. However, tricuspid annular plane systolic excursion decreased significantly. High altitude increased both systolic and diastolic peripheral Blood Pressure (BP) in the total group (SBP: $116.4 \pm 8.9$ vs. $121.4 \pm 10.5, p<0.05$; DBP: 70.7 \pm 6.3 vs $74.7 \pm 8.2, p<0.05$ ), but not the pulse wave velocity.

AMS scores remained modest except for a significantly higher score on the summit day. No significant differences were observed between T1DM subjects and controls in all parameters measured.

Conclusion: Well-trained subjects with T1DM showed similar adaptation of the cardiopulmonary system compared to non-diabetic controls, with increased NTproBNP levels and peripheral blood pressure at high altitude up to $4,000 \mathrm{~m}$ in both groups.

Keywords: NTproBNP; RV function; Pulse wave velocity; Diabetes; High altitude

\section{Introduction}

Hypobaric conditions at high altitude results in hypoxia, which in turn triggers physiological changes in the cardiovascular and pulmonary systems to allow adequate delivery of oxygen to tissues despite the reduced partial pressure of inspired oxygen. Exposure to acute hypoxia leads to mild pulmonary hypertension, increased heart rate, activation of the sympathetic nervous system, and altered vascular resistance [1,2]. Additionally, increases in diastolic blood pressure have also been described to be an adaptation to high altitude [3].

Early signs of pulmonary hypertension include an increase in the diameter of the Right Ventricle (RV) and a decrease in the Tricuspid Annular Plane Systolic Excursion (TAPSE), which is an echocardiographic measure of the RV ejection fraction. Additionally, dilation of the RV can lead to a leftward shift of the interventricular septum which could result in impairment of Left Ventricular (LV) diastolic function. A decrease in RV function may occur as well. Plasma levels of cardiac natriuretic peptides such as Brain Natriuretic Peptide (BNP) and its inactive amino terminal fragment, NTproBNP, serve as biomarkers for cardiac dysfunction. Ventricular wall stress, due to volume expansion and pressure overload, results in an increased synthesis and secretion of BNP and NTproBNP by both ventricles. As such the measurement of cardiac natriuretic peptides, such as BNP and its inactive amino-terminal fragment NTproBNP, is used increasingly in the clinical routine to identify patients with pulmonary hypertension and patients with (suspected) heart failure and RV- and LV dysfunction [2]. When NTproBNP $<50 \mathrm{pg} /$ $\mathrm{ml}$, heart failure is highly unlikely; when NTproBNP $>450 \mathrm{pg} / \mathrm{ml}$, heart failure is likely.

An increasing number of people with a chronic condition, such as patients with Type 1 Diabetes Mellitus (T1DM), ascend 
to high altitudes and expose themselves to hypoxia. During high altitude trekking, the physiological and metabolic adjustments to hypobaric hypoxia are even more pronounced due to the strenuous physical activity involved. Exposure to high altitude conditions is also associated with Acute Mountain Sickness (AMS), which presents as a constellation of signs and symptoms including nausea, fatigue, dizziness, and sleep disturbance which are exacerbated by strenuous activity. Thus, counselling of such patients is now more important than ever [3]. In general, patients with T1DM have an increased cardiovascular mortality and morbidity risk compared to non-diabetic subjects. Many complications in T1DM have a cardiovascular background $[4,5]$. Amongst others, chronic glucose dysregulation is a mediator of cardiovascular risk in these patients, contributing to impaired endothelial and diastolic function, as well as affecting microand macro vasculature. Additionally, a persistent increase in heart rate, which can be seen in a subgroup of patients with diabetes (especially when autonomic neuropathy is present), is thought to contribute to the stiffening of the arteries which is accelerated in both T1DM and Type 2 Diabetes Mellitus (T2DM). Arterial stiffening has been described as an early phenomenon in subjects with T1DM that presents even before the onset of cardiovascular complications. Arterial stiffness itself is associated with increased systolic and pulse pressure [4]. Stiffening of the aorta affects cardiac function by increasing the afterload and reducing coronary artery perfusion during diastole [6]. As such, aortic stiffness represents a risk factor for the development of cardiovascular disease $[7,8]$. Previous studies have shown that subjects with T1DM are able to participate in strenuous forms of exercise such as high altitude trekking and mountaineering [9]. These studies have primarily focused on glycemic control in these subjects. Exercise under conditions of hypobaric hypoxia result in unique challenges on the participants. Among others, altitude can alter glucoregulation, cold temperatures and altitude can complicate accurate reading of the glucose monitoring equipment and storage of insulin. This can lead to either hyperglycemia or hypoglycemia [10]. Exercise, dietary change, stress and illness, increased sympathetic output at altitude can lead to conflicting accounts of insulin requirement [11]. Careful assessment of diabetes-related complications, optimal preparation, and adequate knowledge of glycemic regulation at altitude is much needed [10].

We hypothesized that during high altitude trekking, the combination of hypobaric hypoxia and increased cardiovascular risk in T1DM disproportionately affect cardiac and vascular parameters compared to non-diabetic subjects. We therefore monitored cardiovascular function in patients with T1DM and non-diabetic controls during a trekking expedition to the summit of M. Kilimanjaro, Tanzania (5,895 m).

\section{Methods}

\section{Study population}

Eight patients with type 1 diabetes mellitus (T1DM) and eight non-diabetic control subjects were recruited to participate in the Dutch Diabetes Kilimanjaro Challenge. This expedition was initiated to show that well trained subjects with T1DM but without prior mountaineering experience can safely participate in high altitude climbing. The expedition started with a 4-day acclimatization climb of Mount Meru (4,566 m), one day of rest at $1,300 \mathrm{~m}$, followed by a 7 -day climb of Mount Kilimanjaro (5,895 m). Exclusion criteria were the presence of complications (retinopathy, neuropathy, nephropathy), uncontrolled hypertension (systolic blood pressure > $150 \mathrm{mmHg}$ and/or diastolic blood pressure $>90 \mathrm{mmHg}$ ), severe hypoglycemia unawareness, cardiac disease (abnormal ECG, ICD or pacemaker, cardio-myopathy, coronary or valvular disease), peripheral arterial insufficiency, smoking or use of illicit drugs. All subjects were well trained. A symptom limited cardiopulmonary exercise test was performed in all subjects at sea level and all participants received an individualized training schedule to further improve their fitness. During the time of training, there were multiple training weekends to check the improvement of the participants. Another exercise test was taken shortly before the expedition took place. All participants were informed of the possible risks and gave their voluntary written consent. The study was approved by the medical ethics committee of the Isala Clinics in Zwolle, The Netherlands.

\section{Echocardiography}

Echocardiography assessment was performed at Sea Level (SL) before the expedition and at an altitude of 4,000 $\mathrm{m}$ (2 days prior to the summit ascent) after 10 days acclimatization. All acquisitions were made by an experienced cardiac sonographer using a portable Vivid I ultrasound system (GE Ultrasound) equipped with Tissue Doppler technology (TDI). Care was taken to ensure that the position of the subjects and the transducer were similar in all examinations. The echocardiographic evaluation, according protocol, systematically included Wall Motion Score Index (WMSI, a measure of global LV function), RV diameter, and TAPSE (as a measure of RV ejection fraction). These measurements were obtained in the apical four chamber view. LV systolic and diastolic diameter, septum and posterior wall diameter were measured in the parasternal long axis view.

\section{Assessment of NTproBNP}

NTproBNP at SL was analyzed with an electrochemiluminiscence immunoassay (Elecsys, Roche Diagnostics, Almere, The Netherlands). All blood samples were analyzed immediately after collection. The Elecsys NTproBNP method is unavailable at high altitude, therefore the Cobas h232 (Roche Diagnostics) Point-of-Care (POC) system was used at $4,000 \mathrm{~m}$. The validity of results obtained using this POC system has been evaluated against the Elecsys system and a diagnostic concordance of $93 \%$ was found between the two assays [12]. In analyses of results obtained with the POC assay, values $<60 \mathrm{pg}$ / $\mathrm{ml}$ were replaced by $60 \mathrm{pg} / \mathrm{ml}$ as has been previously reported in the comparison of the two methods [12]. All blood samples were analyzed immediately after being drawn with the subject in supine position.

\section{Vascular measurements}

Vascular measurements were performed in patients with T1DM and control subjects during the expedition at an altitude 
of $1,300 \mathrm{~m}$ and $4,000 \mathrm{~m}$. The subjects were measured in a supine position after a 15 minutes resting period. Five blood pressure measurements were taken with a 2 minute interval using a Dinamap (Mobil-O-Graph®NG). In order to derive the central aortic pressure, pulse wave analysis was performed using a B-PRO, a radial Pulse Wave Acquisition device with possibility to obtain a $24 \mathrm{hr}$ macroscopic view of blood pressure, heart rate, mean arterial pressure and pulse pressure. Pulse wave velocity was assessed three times using a VICORDER [13]. The mean of these 3 measurements was taken as the pulse wave velocity.

\section{Measurement of oxygen saturation}

Oxygen saturation $\left(\mathrm{SatO}_{2}\right)$ was assessed at $4,000 \mathrm{~m}$ by pulse oximetry using Apnea Link with oximetry (Res Med Corp, USA). The oximeter itself stored the measured values and created an average. The average $\mathrm{SatO}_{2}$ was calculated during 30 minutes of rest in an upright position.

\section{Assessment of Acute Mountain Sickness (AMS)}

Participants were required to fill out a daily self-report questionnaire on symptoms associated with development of AMS during the 7-day Kilimanjaro expedition. An AMS score based on the Lake Louise scoring system was calculated. Acetazolamide (250 mg orally) was provided above an altitude of 3,000 $\mathrm{m}$ in order to prevent the onset and/or progression of AMS. The 3,000 m was chosen after the acclimatization period. Medication, besides insulin, was provided only after consultation with one of the doctors and consisted of Paracetamol and Loperamide.

\section{Statistical analyses}

All data are expressed as the mean value \pm SD. The data were analyzed using SPSS software. We used the paired t-test for comparison between values at SL and 4,000 m and the Mann Whitney U test for comparison between the two groups. A p-value of $<0.05$ was considered statistically significant. An ANOVA (GLM repeated measures) analysis was not performed as the power of the test for normality for such a small sample size would be far too low.

\section{Results}

Sixteen subjects, 8 with T1DM (5 male, 3 female) and 8 nondiabetic controls ( 4 male, 4 female) participated in the study. All participants live at SL and had normal renal function. The T1DM cohort had a mean age of $31.5 \pm 5.3$ yrs, diabetes duration of $10.4 \pm 8.1 \mathrm{yrs}$, BMI of $23.5 \pm 1.5 \mathrm{~kg} / \mathrm{m}^{2}$ with a height of $182 \pm$ $12.5 \mathrm{~cm}$ and weight of $78.5 \pm 14.5 \mathrm{~kg}$. They were free from longterm diabetes complications and exhibited good glycemic control (HbA1C $6.8 \pm 0.6 \%$ ). Mean insulin use per day was 45,8 IU/24h at om. Ascending M. Kilimanjaro mean insulin use per day was $43,0 \mathrm{IU} / 24 \mathrm{~h}$ at $1,400 \mathrm{~m} ; 32,4 \mathrm{IU} / 24 \mathrm{~h}$ ascending from $1,500 \mathrm{~m}$ to $2,514 \mathrm{~m} ; 34,1 \mathrm{IU} / 24 \mathrm{~h}$ ascending from $2,514 \mathrm{~m}$ to $3,583 \mathrm{~m}$ and $32,9 \mathrm{IU} / 24 \mathrm{~h}$ ascending from $4,562 \mathrm{~m}$ to the summit and back to $2,514 \mathrm{~m}$. Besides the medication used during the expedition and mentioned before, no further medication was taken. None of the T1DM subjects had previous mountaineering experience, although all were physically fit with a mean exercise capacity of 3.4 Watt/Kg \pm 0.7. Systolic blood pressure (BP) was $139 \pm$
$17 \mathrm{mmHg}$ and diastolic BP $82 \pm 5 \mathrm{mmHg}$, The 8 non-diabetic subjects had a mean age of $43 \pm 10.5$ yrs $(p<0.05)$, BMI was 24 $\pm 1.8 \mathrm{~kg} / \mathrm{m}^{2}$ with a height of $175 \pm 10 \mathrm{~cm}$ and weight of $74 \pm 10$ $\mathrm{kg}$. Average exercise capacity was $3.2 \pm 0.5 \mathrm{Watt} / \mathrm{Kg}$, a systolic BP of $129 \pm 12 \mathrm{mmHg}$ and diastolic BP of $84 \pm 7 \mathrm{mmHg}$. Two out of the 8 control subjects had previous mountaineering experience. All participants climbed to the summit of Mt. Kilimanjaro 5 , 895 $\mathrm{m})$. Our observations on the impact of high altitude trekking on metabolic control have been reported elsewhere [14].

\section{Acute mountain sickness}

Fifteen participants completed their daily AMS score form. AMS scores were modestly elevated at high altitude except for a significantly higher score on day 7 when all participants climbed to the summit of Mt. Kilimanjaro (5,895 m) $(p<0.05)$ (Figure 1A). There were no significant differences in AMS scores between the T1DM and control subjects (Figure 1A). 5/8 T1DM and 4/8 control subjects used acetazolamide (250 mg/ day) given that acetazolamide relieves symptoms of AMS and may therefore impact the reported AMS score, we performed a separate analysis excluding the AMS scores of those subjects who had taken acetazolamide (Figure 1B). With these low sample numbers (3 T1DM, 4 control) no differences could be observed between T1DM and control subjects $(p=0.4)$. At $4000 \mathrm{~m}$, resting oxygen saturation $\left(\mathrm{SpO}_{2}\right)$ was $87 \pm 3 \%$ in the T1DM group and 89 $\pm 2 \%$ in the control subjects $(p=0.2)$.
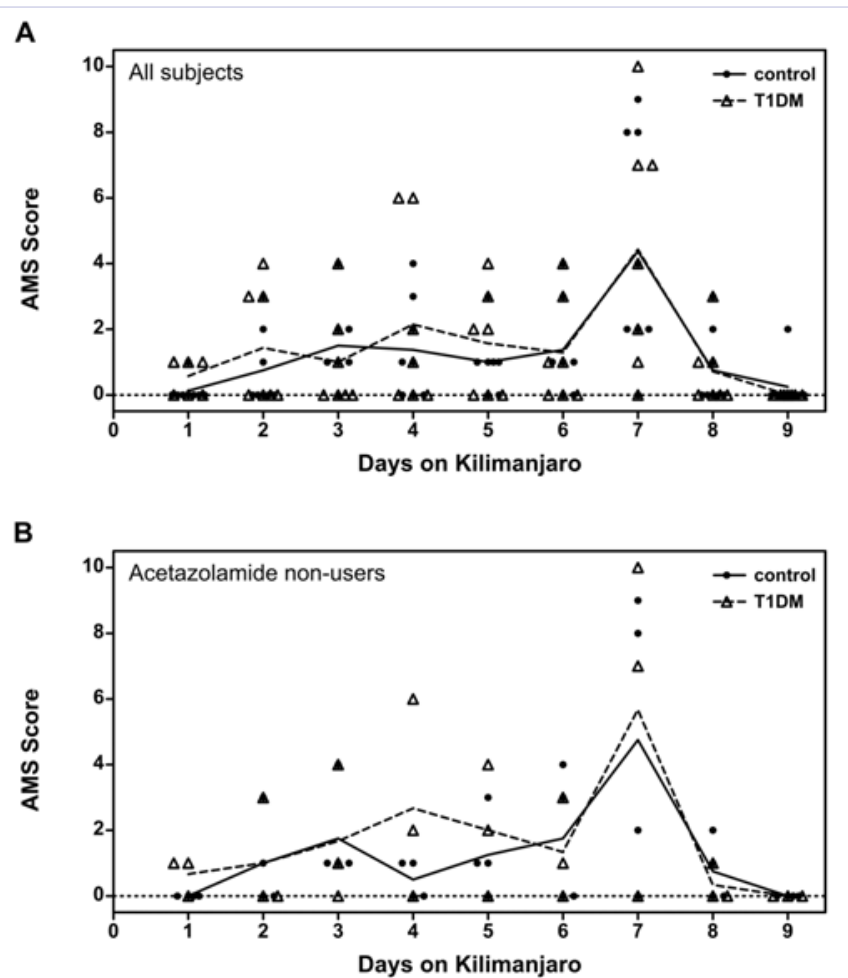

Figure 1: Acute mountain sickness (AMS) scores based on the Lake Louisiana scoring system were obtained daily from 15/16 participants (8 control, 7 type 1 diabetes mellitus, T1DM). (A) AMS scores of all participants. (B) The AMS scores of the acetazolamide non-users only (4 control, 3 T1DM) were plotted and analyzed to exclude and impact of acetazolamide on the differences in AMS score between the two groups. 


\section{Echocardiography}

There were no significant changes in WMSI and RV diameter at high altitude compared to SL in both groups (Table 1). TAPSE decreased significantly in both groups compared to the values obtained at SL $(22.8 \pm 3.8$ at SL vs $20.9 \pm 2.1$ at $4,000 \mathrm{~m}, p<0.05)$, with no significant differences between T1DM and non-diabetic controls. Six subjects (2 T1DM and 4 controls) developed an increase in tricuspid regurgitation at 4,000 m. Estimates of pulmonary artery pressures could not be obtained in all subjects due to technical difficulties.

\section{NTproBNP}

The levels of NTproBNP of each participant measured at SL and at 4,000 m are plotted in Figure 2. NTproBNP levels at rest were significantly higher at an altitude of $4,000 \mathrm{~m}$ compared to sea level $(157 \pm 129$ vs $51 \pm 35 \mathrm{pg} / \mathrm{ml}, p<0.01)$. There were no significant differences between subjects with $(148 \pm 145 \mathrm{pg} / \mathrm{ml})$ and without $(166 \pm 121 \mathrm{pg} / \mathrm{ml}) \mathrm{T} 1 \mathrm{DM}(p=0.3)$ (Table 1). The mean change in NTproBNP levels $\left(\mathrm{NTproBNP}_{4000 \mathrm{~m}}-\mathrm{NTproBNP}_{\mathrm{SL}}\right)$ was similar between acetazolamide users $(107.4 \pm 139.2 \mathrm{pg} / \mathrm{ml})$ and non-users $(105.4 \pm 115.4 \mathrm{pg} / \mathrm{ml})$.

\section{Pulse wave analysis}

High altitude resulted in a significant increase of Systolic Blood Pressure (SBP) in the control group. This group also had a significantly higher Central Aortic Systolic Pressure (CASP) at 4,000 $\mathrm{m}$ than the subjects with T1DM. Pulse wave velocity did not change in either group during this stay at high altitude (Table 1).

\section{Discussion}

In this observational study, all participants with T1DM reached the summit of Mt. Kilimanjaro at an altitude of 5,895 m with similar degrees of arterial hypoxemia compared to the control subjects, thus allowing comparison of cardiovascular parameters between these two groups. The main results of our cardiovascular investigation show that subjects with T1DM who are well trained and exhibit good glycemic control can safely perform high altitude trekking and have a similar cardiac adaptation to hypobaric hypoxia up to 4,000 m compared to equally well trained subjects without diabetes. Our echocardiographic examinations revealed no significant changes in gross cardiac morphology in either the T1DM or non-diabetic subjects. The only significant changes observed were in the TAPSE. TAPSE is a reliable marker of global RV function, and correlates with right ventricular ejection fraction [15]. We found a modest but significant TAPSE decrease in the total group which is indicative of an increase of pulmonary arterial pressure. Indeed, the development of or increase in tricuspid regurgitation in 6 subjects also suggests this. Patients with T1DM were comparable to controls with regard to TAPSE or tricuspid regurgitation.

Both type 1 and type 2 diabetes mellitus may develop increased stiffness of the large arteries which likely contributes to the increased risk of the development of cardiovascular disease in subjects with diabetes. Decreased distensibility results in an increase of the Pulse Wave Velocity (PWV) [6]. Exposure to high altitude is also associated with changes in the cardiovascular function, including altered vascular resistance [1]. Since in theory, subjects with diabetes mellitus would be prone to such changes, we considered it likely that ascending to high altitude might induce an earlier increase in arterial stiffness. However, PWV did not change significantly during this relatively short stay at 4,000 m. High altitude was associated with higher blood pressure, which was more prominent in the control group. Blood pressure is an important contributor to arterial stiffness [4] and may influence PWV. Few studies have been done on vascular measurements at high altitude. Schneider et al. [16] investigated the differences in PWV between recently acclimatized individuals and Himalayan high altitude natives. At baseline there were no differences in PWV at high altitude, however blood flow velocity was higher in the high-altitude native-group following muscular ischemia induced by leg occlusion [16]. Other research at M. Kilimanjaro showed a greater increase in systolic blood pressure in the legs versus the arms of healthy volunteers at an altitude of $4,100 \mathrm{~m}$ probably caused by a higher adrenergic stimulus
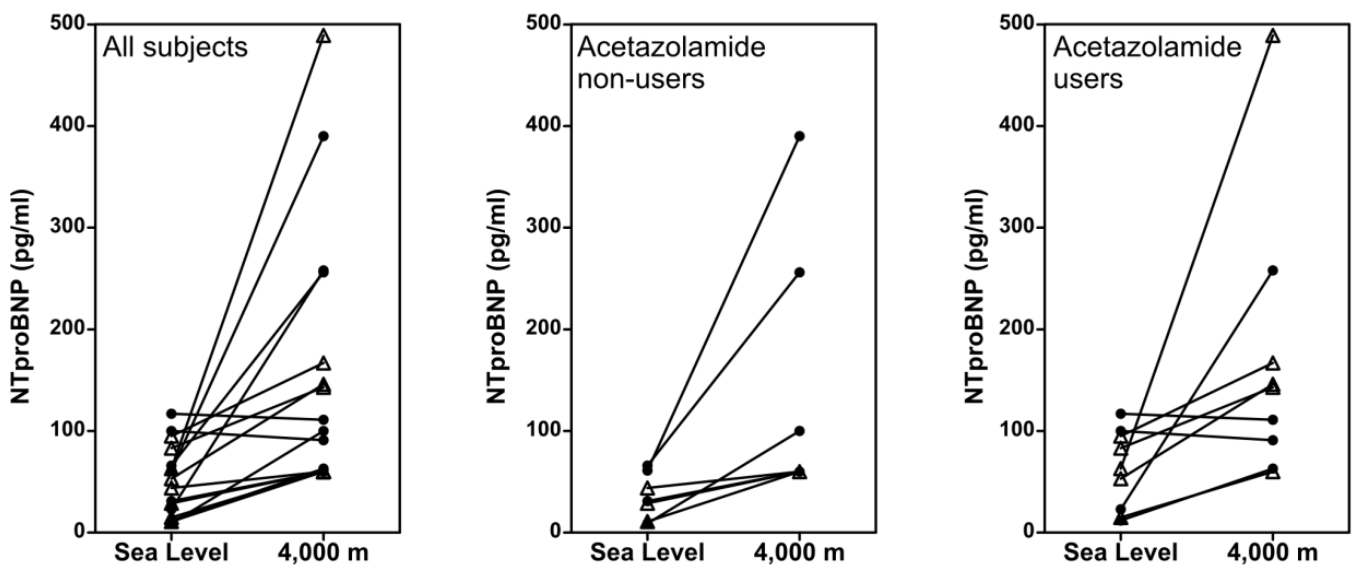

- control

$\Delta$ T1DM

Figure 2: The levels of the inactive amino terminal fragment of Brain Natriuretic Peptide (NTproBNP) of control and type 1 diabetes mellitus (T1DM) subjects were measured at sea level and at $4000 \mathrm{~m}$. Left panel, NTproBNP levels measured for all participants; middle panel, acetazolamide non-users only; right panel, acetazolamide users only. 
Table 1: Echocardiographic and NTproBNP results and Pulse Wave Analysis according to group.

\begin{tabular}{|c|c|c|c|c|}
\hline & T1DM $(\mathrm{N}=8)$ & & Control $(\mathrm{N}=8)$ & \\
\hline & SL & $4,000 \mathrm{~m}$ & SL & $4,000 \mathrm{~m}$ \\
\hline WMSI & $1,0 \pm 0,0$ & $1,0 \pm 0,0$ & $1,0 \pm 0,0$ & $1,0 \pm 0,0$ \\
\hline RV & $43,8 \pm 3,9$ & $42,9 \pm 5,7$ & $40,8 \pm 4,7$ & $41,6 \pm 3,7$ \\
\hline TAPSE & $22,9 \pm 5,2$ & $20,8 \pm 2,8$ & $22,6 \pm 1,9$ & $21,1 \pm 1,3$ \\
\hline LVED (mm) & $48.4 \pm 5.7$ & $47 \pm 11.2$ & $46.9 \pm 5.3$ & $43.9 \pm 10.7$ \\
\hline LVES (mm) & $34.6 \pm 4.7$ & $31.8 \pm 8$ & $32.5 \pm 3.6$ & $32 \pm 7.6$ \\
\hline Septum (,mm) & $6.3 \pm 0.7$ & $6.9 \pm 1.6$ & $7.8 \pm 2.1$ & $7.8 \pm 2.3$ \\
\hline Posterior (mm) & $8.9 \pm 1.6$ & $7.5 \pm 2.1$ & $8 \pm 1.7$ & $8.5 \pm 2.2$ \\
\hline \multirow[t]{2}{*}{ NTproBNP (pg/ml) } & $49 \pm 31^{* * *}$ & $148 \pm 145^{* * *}$ & $52 \pm 41^{*}$ & $166 \pm 121 *$ \\
\hline & $1,300 \mathrm{~m}$ & $4,000 \mathrm{~m}$ & $1,300 \mathrm{~m}$ & $4,000 \mathrm{~m}$ \\
\hline CAPS (mmHg) & $106.9 \pm 4.8$ & $103.7 \pm 6.6 \#$ & $109 \pm 10.1^{*}$ & $113.6 \pm 11.2 \# /^{*}$ \\
\hline SBP (mmHg) & $117.1 \pm 6$ & $119.8 \pm 8.4$ & $115.6 \pm 11.8^{*}$ & $124 \pm 12.8^{*}$ \\
\hline DBP (mmHg) & $68.3 \pm 3.5$ & $71.8 \pm 5.6$ & $73 \pm 8.2$ & $76.4 \pm 9.7$ \\
\hline Mean PWV (m/s) & $5.5 \pm 0.42$ & $5.5 \pm 0.48(\mathrm{n}=7)$ & $5.8 \pm 0.85$ & $6.3 \pm 0.97(n=5)$ \\
\hline
\end{tabular}

WMSI: Wall Motion Core Index; RV: Right Ventricle Diameter; TAPSE: Tricuspid Annular Plane Systolic Excursion; LVED: Left Ventricle End Diastolic Diameter; LVES: Left Ventricle End Systolic Diameter; PWV: Pulse Wave Velocity; CASP: Central Aortic Pressure; SBP: Systolic Blood Pressure; DBP: Diastolic Blood Pressure

${ }^{*}=p<0,05,{ }^{* *}=p<0,01,{ }^{* * *}=p=0,07$ SL $v s 4,000 \mathrm{~m}$ or $1,300 \mathrm{~m}$ vs $4,000 \mathrm{~m}$

$\#=p<0,05 \mathrm{~T} 1 \mathrm{DM} v s$ controls

[17]. A longer stay at high altitude might contribute to further stiffening of the arteries resulting in an increase in PWV, but this phenomenon is not only necessarily associated with the presence of diabetes mellitus. The more prominent increase in blood pressure in control subjects might be explained by the age difference. The mean age of this group was significantly higher than the T1DM group. There is a progressive stiffening of the large arteries with age, which can contribute to the development of systolic hypertension [18].

We demonstrate that NTproBNP levels are increased in both healthy and T1DM subjects at 4,000 m despite an acclimatization to high altitude $(1,800-4,500 \mathrm{~m})$ for 10 days. The increase in NTproBNP could be expected since we found a significant decrease in TAPSE and tricuspid regurgitation developed in several subjects. While our results are congruent with a report by Feddersen et al. [19] who observed an increase in BNP levels at an altitude of 5,050 m in healthy individuals, another study reported no effect of high altitude on NTproBNP levels [20]. The main difference of this study by Toshner et al. [20] to ours was the lack of physical effort by the subjects prior to examination. Thus, while the previous data suggests that hypobaric hypoxia does not impact NTproBNP levels, in our opinion the true conditions of a high altitude expedition which combines hypobaric exposure with strenuous activity were not reflected in the study of Feddersen. Indeed, NTproBNP appears to increase by strenuous exercise [21]. However, given that the half-life of NTproBNP is 60-120 minutes, and that venous blood sampling in our subjects was performed after a mean rest period of 4 hours, it is unlikely that the increase in NTproBNP can be explained by exercise alone. Rather, our results underscore the impact of prolonged exposure to hypobaric conditions combined with daily strenuous activity on NTproBNP levels.
Feddersen et al. [19] also reported a correlation between BNP levels and severity of AMS. We could not observe such a correlation between NTproBNP and AMS scores in our study (data not shown). The Lake Louise Scoring system of AMS symptoms revealed that all participants had a similar susceptibility to AMS symptoms and showed no significant differences in the average daily AMS score reported between T1DM and control subjects, also when corrected for acetazolamide use. However, due to the small numbers of acetazolamide non-users in each group, there was likely insufficient statistical power to detect any differences in this analysis. Acetazolamide was used by several subjects in both groups. Basnyat et al. [22] showed no differences in mean pulmonary artery pressure between groups using acetazolamide and placebo at high altitude. However, the drug was efficacious against AMS. While it is unlikely that the use of acetazolamide had an effect on echocardiographic parameters or NTproBNP concentrations, there is insufficient data at high altitude to completely exclude this possibility.

The small sample size due to the logistic and financial aspects of such a climbing expedition involving subjects with T1DM and no previous climbing experience is a limitation of our study. The non-randomized use of acetazolamide, additionally limits the statistical power of the comparison between the two groups. There is also a significant age difference between both groups, which justifies some cautionary notes in comparing the two. However, despite these limitations, our study shows that well-trained, complication free patients with type 1 diabetes living at sea level and with good glycemic control show similar adaptation of the cardiac system compared to control subjects, with increased NTproBNP concentrations at high altitude in both groups. 


\section{Acknowledgements}

We would like to acknowledge all previous studies mentioned in this manuscript that we could not formally cite due to format restrictions. We like to thank the Bas van de Goor Foundation, The Netherlands, for their support and funding of the expedition. We thank Snow Leopard Travels, The Netherlands, and local porters and guides for expedition logistics and support. We thank Roche Diagnostics for providing the POC system and the Department of Cardiology of the Isala Clinics for the use of the Vivid I (GE Ultrasound). We thank APC cardiovascular for the use of the portable vascular measurement systems. We thank all participants for participation in this study.

\section{References}

1. Naeije R. Physiological adaptation of the cardiovascular system to high altitude. Prog Cardiovasc Dis. 2010; 52(6):456-66. doi: 10.1016/j. pcad.2010.03.004.

2. Daniels LB, Maisel AS. Natriuretic peptides. J Am Coll Cardiol. 2007; 50(25):2357-68.

3. Allemann Y, Scherrer U. High altitude medicine: important for trekkers and mountaineers, essential for the progress in medicine. Prog Cardiovasc Diseases. 2010; 52(6):449-50. doi: 10.1016/j. pcad.2010.02.006.

4. Van Ittersum FJ, Schram MT, van der Heijden-Spek JJ, van Bortel LM, Elte JW, Biemond P, et al. J Hum Hypertens. 2004; 18(11):761-8.

5. Ceriello A, Ihnat MA, Thorpe JE. Clinical review 2: The "metabolic memory": is more than just tight glucose control necessary to prevent diabetic complications? J Clin Endocrinol Metab. 2009; 94(2):410-5 doi: $10.1210 /$ jc.2008-1824.

6. Van Elderen SG, Brandts A, Westenberg JJ, van der Grond J, Tamsma JT, van Buchem MA, et al. Aortic stiffness is associated with cardiac function and cerebral small vessel disease in patients with type 1 diabetes mellitus: assessment by magnetic resonance imaging. Eur Radiol. 2010; 20(5):1132-8. doi: 10.1007/s00330-009-1655-4.

7. Cameron JD, Cruickshank JK. Glucose, insulin, diabetes and mechanisms of arterial dysfunction. Clin Exp Pharmacol Physiol. 2007. 34(7):677-82.

8. Kang S, Fan HM, Li J, Fan LY, Miao AY, Bao Y, et al. (2010). Relationship of arterial stiffness and early mild diastolic heart failure in general middle and aged population. Eur Heart J. 2010; 31(22):2799-807. doi: 10.1093/eurheartj/ehq296.

9. Brubaker PL. Adventure travel and type 1 diabetes. Diabetes Care. 2005; 28(10):2563-72.

10. de Mol P, de Vries, ST, de Koning EJ, Gans RO, Bilo HJ, Tack CJ. (2014). Physical activity at altitude: challenges for people with diabetes: a review. Diabetes Care. 2014. 37(8):2404-13. doi: 10.2337/dc13-2302.

11. Richards $P$, Hillebrandt D. The practical aspects of insulin at high altitude. High Alt Med Biol. 2013; 14(3):197-204. doi: 10.1089/ ham.2013.1020.

12. Alehagen U, Janzon M. A clinician's experience of using the Cardiac Reader NT-proBNP point-of-care assay in a clinical setting. Eur J Heart Fail. 2008; 10(3):260-6. doi: 10.1016/j.ejheart.2008.01.005.

13. Hickson SS, Butlin M, Broad J, Avolio AP, Wilkinson IB, McEniery CM. Validity and repeatability of the Vicorder apparatus: a comparison with the SphygmoCor device. Hypertension Research. 2009. 32(12):1079-85. doi: 10.1038/hr.2009.154.

14. de Mol P, de Vries ST, de Koning EJ, Gans RO, Tack CJ, Bilo HJ. Increased insulin requirements during exercise at very high altitude in type 1 diabetes. Diabetes Care. 2011; 34(3):591-5. doi: 10.2337/dc10-2015.

15. Anjak A, López-Candales A, Lopez FR, Harris D, Elwing J. Objective Measures of Right Ventricular Function during Exercise: results of a pilot study. Echocardiography. 2014; 31(4):508-15. doi: 10.1111/ echo.12417.

16. Schneider A, Greene RE, Keyl C, Bandinelli G, Passino C, Spadacini G, et al. Peripheral arterial vascular function at altitude: sea-level natives versus Himalayan high altitude natives. J Hypertens. 2001; 19(2):21322.

17. Nault P, Halman S, Paradis J. Ankle-brachial index on Kilimanjaro: lessons from high altitude. Wilderness Environ Med. 2009; 20(1):726. doi: 10.1580/08-WEME-BR-186.1.

18. McEniery CM, McDonnell BJ, So A, Aitken S, Bolton CE, Munnery M, et al. Aortic calcification is associated with aortic stiffness and isolated systolic hypertension in healthy individuals. Hypertension. 2009; 53(3): 524-31. doi: 10.1161/HYPERTENSIONAHA.108.126615.

19. Feddersen B, Ausserer H, Haditsch B, Frisch H, Noachtar S, Straube A. Brain natriuretic peptide at altitude: relationship to diuresis, natriuresis, and mountain sickness. Aviat Space and Environ Med. 2009; 80(2):108-11.

20. Toshner MR, Thompson AA, Irving JB, Baillie JK, Morton JJ, Peacock AJ. NT-proBNP does not rise on acute ascent to high altitude. High Alt Med Biol. 2008; 9(4):307-10. doi: 10.1089/ham.2008.1054.

21. Knebel F, Schimke I, Schroeckh S, Peters H, Eddicks S, Schattke S, et al. Myocardial function in older male amateur marathon runners: assessment by tissue doppler echocardiography, speckle tracking, and cardiac biomarkers. J Am Soc Echocardiogr. 2009; 22(7):803-9. doi: 10.1016/j.echo.2009.04.009.

22. Basnyat B, Hargrove J, Holck PS, Srivastav S, Alekh K, Ghimire LV, et al. Acetazoalmide fails to decrease pulmonary artery pressure at high altitude in partially acclimatized humans. High Alt Med Biol. 2008; 9(3):209-16. doi: 10.1089/ham.2007.1073. 